\title{
Development of Learning Objectives for a Medical Assistance in Dying Curriculum for Family Medicine Residency
}

\section{Sarah LeBlanc ( $\sim$ sleblanc@pefht.com )}

Queen's University

\section{Susan MacDonald}

Queen's University

\section{Mary Martin}

Queen's University

Nancy Dalgarno

Queen's University

Karen Schultz

Queen's University

\section{Research Article}

Keywords: Family Medicine, Post-Graduate Medical Education (PGME), Learning Objectives, Euthanasia, Assisted Suicide, Medical Assistance in Dying (MAID)

Posted Date: August 18th, 2021

DOl: https://doi.org/10.21203/rs.3.rs-799267/v1

License: (c) (1) This work is licensed under a Creative Commons Attribution 4.0 International License. Read Full License

Version of Record: A version of this preprint was published at BMC Medical Education on March 11th, 2022. See the published version at https://doi.org/10.1186/s12909-022-03204-1. 


\section{Abstract \\ Background}

Medical assistance in dying (MAID) became legal across Canada when Bill C-14 was passed in 2016. Currently, little is known about the most effective strategies for providing MAID education, and the importance of integrating MAID into existing curricula. In this study, a set of learning objectives (LOs) was developed to inform a foundational MAID curriculum in Canadian Family Medicine (FM) residency training programs.

\section{Methods}

Mixed-methods were used to develop LOs based on a previously-published needs assessment from a large, four-site family medicine residency program in southeastern Ontario. Draft LOs were evaluated and modified using a modified Delphi process and focus group which included faculty and resident leaders. LOs were mapped to the existing family medicine residency curriculum, as well as the College of Family Physicians of Canada's Priority Topics and CanMEDS-Family Medicine roles.

\section{Results}

Nine LOs were developed to provide a foundational education regarding MAID. While all LOs could be mapped to the Domains of Clinical Care with the departmental curriculum, they mapped inconsistently to departmental Entrustable Professional Activities, the Priority Topics, and CanMEDS-FM roles. LOs focused on patient education and identification of patient goals were most readily mapped to existing curricular framework, while LOs with MAID-exclusive content revealed gaps in the current curriculum.

\section{Conclusions}

The developed LOs provide a guide to ensure family medicine residents obtain generalist-level knowledge to counsel their patients about MAID. These LOs can serve as a model for developing learning objectives for both family medicine and specialist residency programs in Canada, as well as globally in countries where assisted dying is legal.

\section{Background}

Discussions regarding medical assistance in dying (MAID) have been in the Canadian public eye since Sue Rodriguez's appeal to the Supreme Court in 1993 [1]. MAID became a Canadian reality in 2015 with the landmark Supreme Court decision in Carter v. Canada and subsequently, when Bill C-14 received royal assent in June, 2016 [2]. The introduction of MAID marked a change in the culture and practice of medicine in Canada. As requests for MAID were being met by family physicians and specialist 
colleagues, medical educators were faced with the challenge of determining how best to educate residents about this new facet of medicine.

Since legalization of MAID, medical students and residents across Canada have been surveyed regarding their interest in providing MAID and their educational needs. The first such survey, published by this group in 2018 , revealed that $37 \%$ of family medicine residents in one Canadian residency program were willing to be part of a team providing MAID by intravenous administration [3]. Similarly, a study of 247 residents from six family medicine training programs in Canada, surveyed in 2016/17 revealed that $52 \%$ of residents were willing to participate in withdrawal of treatment for patients at end of life, while fewer were willing to provide MAID by prescription of a lethal drug (31\%) and or direct administration via intravenous (24\%) [4].

Studies of medical students across Canada reveal a higher proportion of learners willing to take part in assisted dying. A study of undergraduate medical students at one Ontario medical school, published in 2017 , revealed that $88 \%$ of those students supported the Supreme Court's decision to allow MAID, and $61 \%$ believed they would provide the means for a patient to end their own life [5]. A large 1210-participant study of Canadian medical students from 15 of 17 medical schools revealed that $71 \%$ of respondents reported being willing to provide MAID. Regional variability of willingness to participate in MAID was noted, in that medical students born in Québec (82\%) were more willing to provide MAID than respondents from other provinces, and conversely, those from Alberta were least likely (63\%) [6].

Our previous study of family medicine residents revealed that residents felt that it was important to receive education surrounding advanced care planning (93\%), discussion of MAID with patients (92\%), regulations and legal aspects of MAID (90\%), ethical issues (89\%) and a general overview of processes and protocols (82\%) [3]. Medical students from a similar study sought access to MAID-specific training for medicolegal issues (91\%), communication skills (80\%), technical aspects of administering MAID (75\%) and education around the doctrines of different religions (49\%) [5].

The purpose of this study was to develop a set of learning objectives (LOs) to inform the MAID curriculum in Family Medicine Residency Training Programs. LOs are essential components of any curriculum as they focus and guide the intended learning and inform assessment criteria [7]. For the purpose of this paper, LOs are defined as activities that residents will be competent in by the end of their training program. With this publication, these LOs can be adapted for use in residency programs across Canada to deliver a foundational education regarding Medical Assistance in Dying.

\section{Methods}

As part of a larger study aiming to develop curricular content for a Family Medicine residency program, this mixed-methods study builds upon a needs assessment published in 2018 [3] to determine the educational needs of family medicine residents at a large, 4-site family medicine residency program in southeastern Ontario. Here, we describe a four-part process of developing, refining and mapping a set of LOs to curriculum. Our study was reviewed for ethical compliance by Queen's University and the Health 
Sciences and Affiliated Teaching Hospitals Research Ethics Board (TRAQ 6021252). All participants provided informed consent.

\section{Development of LOs}

The initial draft of LOs was developed by the research team (SL, SM, ND) through review of the needs assessment ${ }^{1}$ and a review of relevant literature.

\section{Delphi Process}

Data were collected from a consensus group of six experts. This group consisted of the four Site Directors, a Program Director and Chief Resident in the family medicine program. A modified-Delphi method $[8,9]$ was used and the initial draft LOs were reviewed anonymously using an online survey system (Qualtrics). Participants could choose to approve the LO, delete the LO or modify the LO. After each round, responses were reviewed; LOs that reached $75 \%$ consensus were taken as final, and those that failed to reach consensus were discussed and revised before being submitted to the next Delphi round. A maximum of 3 rounds were agreed upon prior to initiation.

\section{Focus Group}

A key informant focus group was conducted, consisting of the DFM Assessment Director and two FM faculty who are knowledgeable about MAID and the DFM assessment system. The aim was to review the LOs reached by consensus and determine if revision to current assessment tools was required to ensure MAID LOs were achievable. The script was developed by researchers (SL, SM, ND) based on a review of the needs assessment survey [3] as well as results of the Delphi process. The Focus Group was audiorecorded and transcribed verbatim. It was $1.5 \mathrm{~h}$ in length and moderated by one researcher (ND), who is not involved in the delivery of curriculum. Qualitative analysis was carried out on the transcripts by one researcher (MM) to identify themes and to summarize suggested changes to the LOs.

\section{Curriculum Mapping}

The MAID LOs were mapped onto the existing DFM curriculum that includes the program's Domains of Clinical Care and entrustable professional activities (EPAs), as well as the College of Family Physicians of Canada (CFPC) CanMEDS-FM [10] and 105 priority topics[11] documents. Search terms included medical assistance in dying, euthanasia, assisted suicide, assisted death, advanced care planning, conscientious objection/objectors, religion, communication (family, patients, caregivers), long term care, pallative care, and end of life. Any LOs that did not map onto the existing competencies and learning activities highlighted gaps and prompted the development of required MAID-specific curricular content.

\section{Results}

The initial development of LOs based on a review of literature and discussion among researchers resulted in a draft list of 9 LOs (Table 1), which were used in Round 1 of the Delphi process. 
Table 1. Learning Outcomes for the MAID curriculum after Delphi Process

LO1: Define medical assistance in dying (MAID), euthanasia, and assisted suicide.

LO2: Describe the eligibility criteria for medical assistance in dying (MAID) in Canada.

LO3: Recognize which regulatory safeguards exist in Canada's Bill C-14 to protect patients seeking medical assistance in dying (MAID).

LO4: Explain in general terms to patients how medical assistance in dying (MAID) is provided in Canada, both in the hospital and the community.

LO5: Explore with patients their motivations for seeking MAID.

LO6: Provide the information required to obtain valid consent from their patients for medical assistance in dying (MAID).

LO7: Understand the benefits and limitations of advanced care planning related to medical assistance in dying (MAID).

LO8: Develop strategies for speaking about medical assistance in dying (MAID) with patients and their families, and with colleagues.

LO9: Describe ethical elements of medical assistance in dying (MAID).

Demographics were not collected due to the small sample size and potential for identification of expert participants.

Round 1 of the Delphi method had a $100 \%$ response rate $6 / 6$ participated) and an $80 \%$ completion rate $(4 / 6)$. It resulted in consensus for 8 of the 9 LOs at $75 \%$ agreement. Upon reflection and discussion, the research team modified one additional LO that reached $75 \%$ consensus based on written feedback in the Delphi Survey. Round 2 included 2 LOs, and had a response rate of $80 \%(4 / 6)$ and was completion rate of $(100.0 \%)$. It resulted in $75 \%$ consensus for the last 2 LOs. Therefore, only two rounds were required.

Results are summarized in Table 2. 
Table 2

Results of Delphi Rounds 1 and 2

\begin{tabular}{|lll|}
\hline LO & \% Consensus Round 1 & \% Consensus Round 2 \\
\hline 1 & $100 \%$ & N/A \\
\hline 2 & $100 \%$ & N/A \\
\hline 3 & $100 \%$ & N/A \\
\hline 4 & $50 \%$ & $75 \%$ \\
\hline 5 & $75 \%$ & N/A \\
\hline 6 & $100 \%$ & N/A \\
\hline 7 & $75 \%$ & $75 \%$ \\
\hline 8 & $100 \%$ & N/A \\
\hline 9 & $100 \%$ & N/A \\
\hline
\end{tabular}

\section{Focus Group}

The focus group resulted in further refinement of 4 of the 9 LOs $(3,5,7$ and 9$)$ in order to ensure LOs were achievable given the current assessment process. Table 3 lists the final LOs.

Table 3. Final Learning Outcomes for the MAID curriculum

LO1: Define medical assistance in dying (MAID), euthanasia, and assisted suicide.

LO2: Describe the eligibility criteria for medical assistance in dying (MAID) in Canada.

LO3: Describe which regulatory safeguards exist in Canada's Bill C-14 to protect patients seeking medical assistance in dying (MAID).

LO4: Explain in general terms to patients how medical assistance in dying (MAID) is provided in Canada, both in the hospital and the community.

LO5: Identify patient motivations for seeking MAID.

LO6: Provide the information required to obtain valid consent from their patients for medical assistance in dying (MAID).

LO7: Describe the benefits and limitations of advanced care planning related to medical assistance in dying (MAID).

LO8: Develop strategies for speaking about medical assistance in dying (MAID) with patients and their families, and with colleagues.

LO9: Describe ethical complexities associated with medical assistance in dying (MAID) for both patients and physicians.

Thematic analysis of discussion resulted in three major themes: Assessing competence, curricular content and delivery, and making curricular changes.

\section{Assessing competence}


Participants agreed that it wasn't feasible to assess every objective for every resident, "We don't have an assessment outcome for every objective we have. We have thousands of objectives." Rather, it was noted that as long as the LOs can be mapped to an EPA, the general skill would be assessed in some way.

\section{Curricular Content and Delivery}

In terms of overall objectives and what they should encompass, participants agreed that residents need to be able to identify, on a fundamental level, a simple MAID case and a complex MAID case - and know when and how to get help when they need it. Furthermore, they agreed on the importance of defining and acknowledging what knowledge of MAID would be useful for a generalist (i.e. a Primary Care Provider), compared to a MAID specialist. In terms of delivery, participants suggested that MAID curriculum be presented in a Module for self-assessment within the end-of-life unit.

\section{Making Curricular Changes}

Participants agreed discussion around changes made to curriculum would need to occur at the level of the Domains of Clinical Care Committee, and is largely out of the control at the local-level. They agreed that the topic area of "End of Life Care" would be the most appropriate curricular area for these discussions to take place.

\section{Curriculum Mapping}

Each LO was mapped to the Domains of Clinical Care (DOCC) within the existing Department of Family Medicine (DFM) curricular framework. The components of the curriculum to which the LOs were mapped were the Domains of Clinical Care (DOCC) within the DFM curriculum, the Entrustable Professional Activities (EPAs), the 105 Priority Topics from the College of Family Physicians of Canada [11], and the CanMEDS-FM [10]. This analysis revealed that while all of the LOs could be mapped to the DOCC, the LOs mapped inconsistently to existing EPAs, 105 Priority Topics, and CanMEDS-FM. LOs 5, 6, and 8 mapped onto the 105 priority topics; LOs 5 and 8 mapped onto the EPAs, and no LOs mapped onto the CanMEDSFM.

The LOs that centred around patient education and identification of patient goals (LOs 5, 6 and 8) were most readily mapped to the curricular framework. LOs with MAID-exclusive content (LOs 1, 2, 3, 4, 7, 9) revealed gaps in the current curricular framework.

\section{Discussion}

In this project, we developed and refined a resultant set of LOs for MAID Family Medicine Residency Education using a Delphi process and a focus group of key experts. LOs are an important component in integrating a MAID focused curriculum as it guides teaching and learning activities, resources, and assessment strategies, documents the competencies residents are to acquire, and informs how student learning is measured [7]. The LOs provide a guide for resident learning with the aim of ensuring FM Residents obtain a generalist - level of knowledge and skills required to identify, counsel and assess 
patients for MAID eligibility. These LOs can be used not only to develop FM MAID curriculum and assess competency through inclusion in EPAs, but also serve as a model for developing LOs and curriculum for other specialty residency programs in Canada.

Previous studies have underlined the willingness and interest of Canadian medical students and residents in taking part in MAID discussions with their patients $[3,4,12,13]$ and the growing importance of MAID education for FM residents, as the system for assessment and provision of MAID in Ontario relies heavily on this specialty [14]. Despite this, there remains a gap in curriculum content, delivery as well as evaluation and assessment of the knowledge and skills required of generalist MAiD assessors.

A Canadian study by Downar et. al. [15] describes the development of an EPA Descriptor for MAID assessors. While the current study focuses on a more generalist approach to assessing and counselling patients and family on MAID, Downar et. al. provide a detailed list of the knowledge and skills required of MAID specialists to not only assess for MAiD, but also technical aspects involved in the provision of MAID. Nonetheless, all of the LOs developed in this study align with those identified by Donwar et al. Shared findings of required knowledge includes eligibility criteria (LO2), regulatory safeguards (LO3), ethical considerations and controversy (LO9) and in general, how MAID is provided (LO4). Skills that align in both studies include assessing eligibility and valid consent (LO6), identifying why a patient would choose MAID (LO5), discussing alternate treatments and end of life planning (LO7), strategies for discussing aspects of MAID with patients and family (LO8).

These have begun to be integrated into the DFM curriculum and next steps can include a needs assessment for Faculty Development (FD), as well as delivery of FD to ensure consistent teaching of the developed MAID-related learning outcomes. The next step of the evaluation of the curriculum will be to conduct a post-test to assess changing clinical behaviour (Level 3 of the Kirkpatrick Model) through inclusion of MAID into the existing Entrustable Professional Activities (EPAs) within the curricular framework.

\section{Limitations}

Our study was conducted at one Family Medicine Residency program in South Eastern Ontario, which limits generalizability to other contexts. Further, though we adopted a Dillman design with multiple reminders, only $4 / 6$ members $(66.7 \%)$ of our expert panel responded to the Delphi design, which was a limitation in this study. As such, this may have led to selection bias, as only those interested in the topic may have been recruited.

\section{Conclusions}

Currently, little is known about the most effective strategies for providing MAID education, and the importance of integrating medical assistance in dying into existing curricula. The purpose of this study was to develop a set of LOs to inform a foundational MAID curriculum in family medicine residency 
training programs. The developed learning objectives provide a guide to ensure family medicine residents obtain generalist-level knowledge to counsel their patients about medical assistance in dying. These learning objectives can be used not only to develop a family medicine MAID curriculum and assess competency, but also serve as a model for developing LOs and curriculum in other specialty residency programs globally.

\section{Abbreviations}

CFPC College of Family Physicians of Canada

DFM Department of Family Medicine

DOCC Domains of Clinical Care

EPA entrustable professional activities

FD Faculty Development

FM family medicine

LO learning objective

MAID Medical assistance in dying

\section{Declarations}

\section{Acknowledgements}

This research was funded through a Janus Grant from the Foundation for Advancing Family Medicine through the College of Family Physicians of Canada, as well as a Research Initiation Grant through the Centre for Studies in Primary Care, Department of Family Medicine, Queen's University.

\section{Ethics approval and Consent to Participate}

Research methods were performed in accordance with the relevant guidelines and regulations of the Declaration of Helsinki. Ethics approval was obtained from the Queen's University and Affiliated Teaching Hospitals Research Ethics Board (file no. 6021390). Written informed consent was obtained from all participants.

\section{Consent for Publication}

The written consent form signed by all participants outlined that the data would be published in a peerreviewed journal.

\section{Availability of data and materials}


Data sharing not applicable to this article as no datasets were generated or analysed during the current study.

\section{Competing Interests}

All of the authors report a Janus Grant from the Foundation for Advancing Family Medicine (FAFM) through the College of Family Physicians of Canada, as well as a Research Initiation Grant through the Centre for Studies in Primary Care, Department of Family Medicine, Queen's University.

\section{Funding}

This research was funded by the College of Family Physicians of Canada as well as the Department of Family Medicine, Queen's University. Neither funding agency had a role in the study's design, conduct or reporting.

\section{Author's contributions}

SL, SM, ND, MM, and KS were equally involved in all aspects of this manuscript. All authors read and approved the final manuscript.

\section{References}

1. Rodriguez v. British Columbia (Attorney General), 1993 < https://scc-csc.lexum.com/scc-csc/scccsc/en/item/1054/index.do>. [accessed 5 June 2016].

2. Department of Justice Government of Canada, 'Legislative Background: Medical Assistance in Dying', 2016 < http://www.justice.gc.ca/eng/rp-pr/other-autre/ad-am/> [accessed 5 June 2016].

3. MacDonald S, LeBlanc S, Dalgarno N, Schultz K, Johnston E, Martin M, Zimmerman D. Exploring family medicine preceptor and resident perceptions of medical assistance in dying and desires for education. Canadian Family Physician. 2018 Sep 1;64(9):e400-6.

4. Wong A, Hsu AT, Tanuseputro P. Assessing attitudes towards medical assisted dying in Canadian family medicine residents: a cross-sectional study. BMC medical ethics. 2019 Dec;20(1):1-8.

5. Bator EX, Philpott B, Costa AP. This moral coil: a cross-sectional survey of Canadian medical student attitudes toward medical assistance in dying. BMC medical ethics. 2017 Dec;18(1):1-7.

6. Falconer J, Couture F, Demir KK, Lang M, Shefman Z, Woo M. Perceptions and intentions toward medical assistance in dying among Canadian medical students. BMC medical ethics. 2019 Dec;20(1):1-7.

7. Sherbino J. Educational design: a CanMEDS guide for the health professions. Royal College of Physicians and Surgeons of Canada; 2011.

8. Dalkey N, Helmer O. An experimental application of the Delphi method to the use of experts. Management science. 1963 Apr;9(3):458-67. 
9. Hsu CC, Sandford BA. The Delphi technique: making sense of consensus. Practical Assessment, Research, and Evaluation. 2007;12(1):10.

10. Shaw E, Oandasan I, Fowler N, eds. CanMEDS-FM 2017: A competency framework for family physicians across the continuum. Mississauga, ON: The College of Family Physicians of Canada; 2017.

11. Crichton T, Schultz K, Lawrence K, Donoff M, Laughlin T, Brailovsky C, Bethune C, van der Goes T, Dhillon K, Pélissier-Simard L, Ross S, Hawrylyshyn S, Potter M. Assessment Objectives for Certification in Family Medicine. Mississauga, ON: College of Family Physicians of Canada; 2020

12. Thomas JM, O'Leary JR, Fried TR. A comparison of the willingness of resident and attending physicians to comply with the requests of patients at the end of life. Journal of general internal medicine. 2014 Jul 1;29(7):1048-54.

13. Bushwick B, Emrhein D, Peters K. A comparison of resident and faculty attitudes toward physicianassisted suicide and active voluntary euthanasia. Fam Med. 2000 Apr 1;32(4):261-6.

14. Health Canada. First Annual Report on Medical Assistance in Dying in Canada. 2019 https://www.canada.ca/en/health-canada/services/medical-assistance-dying-annual-report2019.html\#a5.3. Accessed 15 November 2020.

15. Downar J, Green S, Radhakrishnan A, Wales J, Kim G, Seccareccia D, Wiebe K, Myers J, Kawaguchi S. An entrustable professional activity descriptor for medical aid in dying: a mixed-methods study. CMAJ open. 2018 Oct;6(4):E657. 